\title{
Bridging the gap between epigenetics research and nutritional public health interventions
}

\author{
Graham C Burdge ${ }^{1 *}$ and Karen A Lillycrop ${ }^{2}$
}

\begin{abstract}
Epigenetic processes, primarily DNA methylation and covalent modifications of histones, regulate the transcriptional activity of genes in a manner that can be modified by environmental cues. This allows variation in the expression of the transcriptome without changes in the genome. Constraint in the early life environment, such as poor early nutrition, is associated with increased risk of non-communicable diseases, including cardio-metabolic disease and cancer in later life. Such induced phenotypic change involves environmental signals acting through developmental plasticity. Recent studies in humans and in animal models show that epigenetic processes, in particular DNA methylation, have a central role in the induction and stability of novel phenotypes and in increased disease risk. Identification of such processes suggests the potential for developing biomarkers of disease risk and for interventions to prevent or reverse the adverse effects of a poor early life environment. At present, knowledge in this area is limited to proof-of-principle studies in animal models and some initial studies in humans. Before such findings can be translated into reliable biomarkers and safe, effective interventions, several fundamental questions need to be answered. In order to achieve this, new technologies will be needed to support large cohort studies. Despite the early stage of knowledge in this field and the intellectual, technological and financial challenges, epigenetic research has substantial potential for public health benefits.
\end{abstract}

\section{Epigenetic mechanisms}

The term 'epigenetic' describes a group of heritable, distinct, highly interrelated processes that control the level of mRNA [1]. The main processes are DNA methylation and covalent modifications to the amino termini of histones, principally methylation, acetylation, ubiquitination and phosphorylation. Epigenetic control of mRNA levels can also be conferred through the action of small interfering mRNAs. Epigenetic regulation by DNA methylation is primarily mediated by the addition of $\mathrm{CH}_{3}$ to carbon 5 of cytosines in $\mathrm{CpG}$ dinucleotide pairs that cluster in the 5' regulatory regions of genes, known as CpG islands [1], although other modifications have been described. In general, methylated DNA sequences are associated with gene silencing, whereas unmethylated CpG islands are associated with transcriptional activity. However, methylation of individual CpG dinucleotides allows graded control of transcription through differential changes in the binding of transcription factors and other proteins. Thus, methylation of a CpG that influences a response element for an inhibitory transcription factor could enhance the level of transcription.

The genome is rapidly demethylated in early embryos, with the exception of parentally imprinted genes. This is followed by gene-specific patterns of DNA silencing, which are fundamental to cell differentiation and which are induced de novo by the activities of DNA methyltransferases (Dnmts) $3 \mathrm{a}$ and $3 \mathrm{~b}$, possibly in association with Polycomb proteins. Patterns of methylated cytosines are maintained during DNA replication by Dnmt-1, which uses the hemimethylated DNA as a template.

It is generally thought that patterns of DNA methylation induced during early development are stable throughout the life course. However, a recent study [2] of the level of methylation of individual CpG dinucleotides in the serotonin transporter gene in buccal cells from monozygotic and dizygotic twins collected at 5 and 10 years has shown that in $32 \%$ of the children studied, there was a $5 \%$ difference in methylation, and $14 \%$ of children had a difference of at least $10 \%$ over 5 years (overall range for the whole group $-41 \%$ to $+23 \%)$. One possible explanation for such variation could lie in the proposed dynamic nature of DNA methylation. Szyf [3] has
IInstitute of Human Nutrition, University of Southampton School of Medicine, Institute of Developmental Sciences Building (MP887), Southampton General Hospital, Tremona Road, Southampton, SO16 6YD, UK

Full list of author information is available at the end of the article 
proposed that rather than cytosine methylation being a stable modification, the level at any one time represents the equilibrium between the activities of Dnmts and demethylases such that a shift in the activities of these enzymes could alter the level of methylation even in nondividing cells. If correct, this could have important implications for understanding the epigenetic effects described below.

Epigenetic control of transcription by histone modifications might be regarded as more dynamic than control by DNA methylation. However, the two are closely linked through the binding of specific proteins, such as methyl CpG binding proteins and methyl domain binding proteins, to methylated DNA sequences, which, in turn, recruit histone modifying enzymes [1], and through the recruitment of Dnmts to DNA by histone deacetylase-1 and the Polycomb protein EZH2 [4,5]. In general, acetylation of histones is associated with transcriptionally active euchromatin, whereas removal of acetyl groups by histone deacetylases and methylation of specific amino acid residues by histone methyl transferases induces compact, transcriptionally silenced heterochromatin.

Epigenetic processes can be modified by environmental signals and therefore provide a mechanism by which the expression level of a gene can be adjusted in order to facilitate cellular response. There is emerging evidence that modifications in epigenetic processes may be an important causal factor in disease risk throughout the life course.

\section{The early life environment and future disease risk}

Constraint in the early life environment, such as poor nutrition and endocrine factors, induces a life-long increase in the risk of non-communicable diseases, including cardio-metabolic disease, affective disorders, osteoporosis and cancer [6]. This involves an induced change in the phenotype of the offspring by environmental cues that act through developmental plasticity. The induced phenotype change may represent adaptations that predict the future environment but that result in disease if the prediction is incorrect [7]. Such phenotypic changes involve altered epigenetic regulation of specific genes.

So far, the majority of reports in this area have been proof-of-principle studies in experimental models, although there have been some recent reports on the effect of early life environment on epigenetic processes in humans. Uterine artery ligation in rats induced decreased methylation and increased expression of the $p 53$ promoter in the kidneys of the offspring, which was associated with impaired development of kidney structure [8]. Poor maternal nursing in rats was associated with increased methylation of a CpG in the glucocorticoid receptor $(G R)$ gene in the hippocampus in the offspring, which impaired binding of nerve growth factor 1-A to its receptor and was associated with poor stress response in adulthood [9]. Altered methylation was reversed by cross-fostering the offspring after birth or by infusion of a histone deacetylase inhibitor [9]. Hypermethylation of a CpG in a comparable region of the GR promoter in human brain was also found in individuals with a history of childhood abuse who subsequently committed suicide [10]. These studies [8-10] have important implications for understanding how the early life environment can affect future patterns of behavior and for susceptibility to mental illness.

The quality of nutrition during development is an important causal factor in future disease risk in humans, in particular cardiovascular disease and metabolic syndrome [6]. In Agouti viable yellow ( $A v y)$ mice, increased intake of methyl donors and folic acid induced a graded switch in coat color by inducing increased methylation of a retrotransposon containing a cryptic promoter located proximal to the Agouti gene [11]. In rats, neonatal overfeeding due to small litter size induced hypermethylation of the hypothalamic insulin receptor and proopiomelanocortin promoters [12,13]. Feeding a protein-restricted (PR) diet to pregnant rats induced hypomethylation of and increased mRNA expression from the promoters of the $G R$ and peroxisome proliferator-activated receptor $\alpha$ $(P P A R \alpha)$ genes in the liver of the offspring [14]. Hypomethylation of the $G R$ promoter was accompanied by an increased level of the histone modifications normally associated with active transcription, and with decreased expression of Dnmt1 and lower levels of binding of Dnmt1 to the GR promoter [15]. This suggested that induction of hypomethylation of $G R$ may involve impaired capacity for maintaining methylation patterns during cell replication. Analysis of individual CpGs in the liver PPAR $\alpha$ promoter showed that four CpGs were hypomethylated in the offspring of dams fed a PR diet; two of these CpGs predicted the level of transcription [16].

Supplementation of the maternal PR diet with folic acid prevented the induction of an altered phenotype and of hypomethylation of the GR and PPAR $\alpha$ promoters [14]. However, within the PPAR $\alpha$ promoter, two CpGs that were unaffected by the PR diet alone were hypermethylated in the offspring of dams fed the folic acid supplemented diet [16]. Thus, although folic acid supplementation seemed to prevent the induction of an altered phenotype and epigenotype, this intervention may have induced a vulnerability in the regulation of this gene, and potentially of others. This is supported by the finding that the maternal PR diet induced a persistent change in 311 genes in the liver of the adult offspring, whereas the folic acid supplemented PR diet altered the mRNA expression of 191 [17]. However, only 16 genes were altered in both groups of offspring [17]. Furthermore, folic acid supplementation of the offspring of rats fed a control or 
PR diet during pregnancy during their juvenile-pubertal period induced dyslipidemia and hepatosteatosis (fatty liver) irrespective of maternal diet, and this was accompanied by hypermethylation of the liver PPAR $\alpha$ promoter and hypermethylation of the insulin receptor in adipose tissue [18]. Despite the deleterious effects of folic acid supplementation, these findings show that epigenetic plasticity extends beyond the period of early development and that the epigenetic regulation of specific genes can be modified by dietary interventions in free living offspring.

Two recent studies [19-21] have reported the effect of prenatal under-nutrition on promoter methylation in humans. Individuals who were in utero during the 194445 famine in the Netherlands show an increased risk of cardio-metabolic disease compared with unexposed individuals [19]. The precise pattern of disease is contingent on the timing during development of exposure to famine. Adults who were exposed to famine in utero showed altered DNA methylation in the promoters of several imprinted and non-imprinted genes in white blood cells $[20,21]$. However, the difference between exposed and unexposed individuals was small (around 5\%) compared with that in the GR promoter of childhood abuse victims [9] (about 30\%), which makes the biological significance of findings of these studies difficult to interpret.

\section{Translation of knowledge about epigenetic processes into public health policy}

The identification of a role for epigenetic processes in differential risk of non-communicable diseases is an important step towards interventions to prevent or reverse risk of these conditions. Given that cardio-metabolic disease and cancer develop subclinically throughout the life course, such knowledge could also be applied to the development of epigenetic markers of disease risk before the clinical phase of the condition or to monitor the efficacy of an intervention.

In order to translate knowledge about epigenetics for use in a healthcare or public health context, several key issues need to be addressed. Which epigenetic marks should be targeted and in which genes? What is the variation in these epigenetic marks in the general population and how does this equate to disease risk? Are there tissues in which epigenetic marks can act as a proxy for those in less accessible, but clinically important, tissues? The following additional questions also need to be considered. Given that the same treatment can induce opposite effects in different genes within the same tissue, how can interventions be targeted to produce benefit without also inducing vulnerability in gene regulation, which could increase disease risk? How plastic is the epigenome during the life course? When are epigenetic biomarkers likely to be of use and when are interventions likely to be most effective? How do epigenetic and genetic variation interact?

Answering these questions is a major challenge, not least in terms of financial investment. Furthermore, new technologies, such as methods for rapid sequencing of differentially methylated regions of the genome, need to be developed before large-scale epidemiological studies can be conducted.

\section{Conclusions}

At present, because of the relative novelty of the field, the challenges that need to be overcome in order to develop useful biomarkers and safe and effective interventions are substantial. Nevertheless, the potential benefits from research in this area are likely to be considerable.

\section{Abbreviations}

Dnmt, DNA methyltransferase; GR, glucocorticoid receptor; PPARa, peroxisome proliferator-activated receptor a; PR, protein-restricted.

\section{Competing interests}

The authors declare that they have no competing interests.

\section{Authors' contributions}

GCB and KAL contributed equally to the planning and writing of this manuscript. Both authors read and approved the final manuscript.

\section{Acknowledgements}

Research in the authors' laboratory is funded by the British Heart Foundation and the Biotechnology and Biological Sciences Research Council.

\section{Author details}

'Institute of Human Nutrition, University of Southampton School of Medicine, Institute of Developmental Sciences Building (MP887), Southampton General Hospital, Tremona Road, Southampton, SO16 6YD, UK. '2Development and Cell Biology, University of Southampton School of Biological Sciences, Institute of Developmental Sciences Building (MP887), Southampton General Hospital, Tremona Road, Southampton, SO16 6YD, UK.

Published: 5 November 2010

\section{References}

1. Goldberg AD, Allis CD, Bernstein E: Epigenetics: a landscape takes shape. Cell 2007, 128:635-638.

2. Wong CCY, Caspi A, Williams B, Craig IW, Houts, R, Ambler A, Moffitt TE, Mill J: A longitudinal study of epigenetic variation in twins. Epigenetics 2010, 5:516-526.

3. Szyf M: The dynamic epigenome and its implications in toxicology. Toxicol Sci 2007, 100:7-23.

4. Rountree MR, Bachman KE, Baylin SB: DNMT1 binds HDAC2 and a new co-repressor, DMAP1, to form a complex at replication foci. Nat Genet 2000, 25:269-277.

5. Viré E, Brenner C, Deplus R, Blanchon L, Fraga M, Didelot C, Morey L, Van Eynde A, Bernard D, Vanderwinden JM, Bollen M, Esteller M, Di Croce L, de Launoit Y, Fuks F: The Polycomb group protein EZH2 directly controls DNA methylation. Nature 2006, 439:871-874.

6. Gluckman PD, Hanson MA, Cooper C, Thornburg KL: Effect of in utero and early-life conditions on adult health and disease. N Engl J Med 2008, 359:61-73.

7. Gluckman PD, Hanson MA: Living with the past: evolution, development, and patterns of disease. Science 2004, 305:1733-1736.

8. Pham TD, MacLennan NK, Chiu CT, Laksana GS, Hsu JL, Lane RH: Uteroplacental insufficiency increases apoptosis and alters p53 gene methylation in the full-term IUGR rat kidney. Am J Physiol Regul Integr Comp Physiol 2003, 285:R962-R970.

9. Weaver IC, Cervoni N, Champagne FA, D'Alessio AC, Sharma S, Seckl JR, 
Dymov S, Szyf M, Meaney MJ: Epigenetic programming by maternal behavior. Nat Neurosci 2004, 7:847-854.

10. McGowan PO, Sasaki A, Huang TC, Unterberger A, Suderman M, Ernst C, Meaney MJ, Turecki G, Szyf M: Promoter-wide hypermethylation of the ribosomal RNA gene promoter in the suicide brain. PLOS ONE 2008, 3:e2085.

11. Waterland RA, Jirtle RL: Transposable elements: targets for early nutritional effects on epigenetic gene regulation. Mol Cell Biol 2003, 23:5293-5300.

12. Plagemann A, Harder T, Brunn M, Harder A, Roepke K, Wittrock-Staar M, Ziska T, Schellong K, Rodekamp E, Melchior K, Dudenhausen JW: Hypothalamic proopiomelanocortin promoter methylation becomes altered by early overfeeding: an epigenetic model of obesity and the metabolic syndrome. J Physiol 2009, 587:4963-4976.

13. Plagemann A, Roepke K, Harder T, Brunn M, Harder A, Wittrock-Staar M, Ziska T, Schellong K, Rodekamp E, Melchior K, Dudenhausen JW: Epigenetic malprogramming of the insulin receptor promoter due to developmental overfeeding. J Perinat Med 2010, 38:393-400.

14. Lillycrop KA, Phillips ES, Jackson AA, Hanson MA, Burdge GC: Dietary protein restriction of pregnant rats induces and folic acid supplementation prevents epigenetic modification of hepatic gene expression in the offspring. J Nutr 2005, 135:1382-1386.

15. Lillycrop KA, Slater-Jefferies JL, Hanson MA, Godfrey KM, Jackson AA, Burdge GC: Induction of altered epigenetic regulation of the hepatic glucocorticoid receptor in the offspring of rats fed a protein-restricted diet during pregnancy suggests that reduced DNA methyltransferase-1 expression is involved in impaired DNA methylation and changes in histone modifications. Br J Nutr 2007, 97:1064-1073.

16. Lillycrop KA, Phillips ES, Torrens C, Hanson MA, Jackson AA, Burdge GC:
Feeding pregnant rats a protein-restricted diet persistently alters the methylation of specific cytosines in the hepatic PPARalpha promoter of the offspring. Br J Nutr 2008, 100:278-282.

17. Lillycrop KA, Rodford J, Garratt ES, Slater-Jefferies JL, Godfrey KM, Gluckman PD, Hanson MA, Burdge GC: Maternal protein restriction with or without folic acid supplementation during pregnancy alters the hepatic transcriptome in adult male rats. Br J Nutr 2010, 103:1711-1719.

18. Burdge GC, Lillycrop KA, Phillips ES, Slater-Jefferies JL, Jackson AA, Hanson MA: Folic acid supplementation during the juvenile-pubertal period in rats modifies the phenotype and epigenotype induced by prenatal nutrition. J Nutr 2009, 139:1054-1060.

19. Roseboom T, de RS, Painter R: The Dutch famine and its long-term consequences for adult health. Early Hum Dev 2006, 82:485-491.

20. Heijmans BT, Tobi EW, Stein AD, Putter H, Blauw GJ, Susser ES, Slagboom PE, Lumey LH: Persistent epigenetic differences associated with prenatal exposure to famine in humans. Proc Natl Acad Sci USA 2008, 105:17046-17049.

21. Tobi EW, Lumey LH, Talens RP, Kremer D, Putter H, Stein AD, Slagboom PE, Heijmans BT: DNA methylation differences after exposure to prenatal famine are common and timing- and sex-specific. Hum Mol Genet 2009, 18:4046-4053.

doi:10.1186/gm201

Cite this article as: Burdge GC, Lillycrop KA: Bridging the gap between epigenetics research and nutritional public health interventions. Genome Medicine 2010, 2:80 